\title{
Inductive properties of fixed point sets of mappings on posets and on partially ordered topological spaces
}

Jinlu Li*

"Correspondence: jli@shawnee.edu

Department of Mathematics,

Shawnee State University,

Portsmouth, Ohio 45662, USA

\section{Springer}

\begin{abstract}
In this paper, we prove several fixed point theorems on both posets and partially ordered topological spaces for set-valued mappings. We also provide the inductive properties of the sets of fixed points for some order-increasing mappings.
\end{abstract}

MSC: 06A06; 06F30; 47H04

Keywords: poset; partially ordered topological space; fixed point; order-preserving mapping

\section{Introduction}

Fixed point theory has been developed for more than a century and has become an important branch in mathematics. It has been widely applied to many branches in pure mathematics and applied mathematics, such as: differential equations; integral equations; optimization theory; game theory; economics theory (see $[1,2])$. Fixed point theorems have played important roles to prove the solvability of equations, the existence of solutions for some optimization problems and the existence of Pareto and Nash equilibriums in game theory.

Under similar circumstances, fixed point theorems on posets have become very significant tools in solving some problems with partially ordered underlying spaces. For example, they have been applied to solve equilibrium problems for strategic games with incomplete preferences (see [3]), ordered optimization problems and ordered variational inequality problems (see [4]), nonlinear Hammerstein integral equations (see [5]) and ordinal differential equations (see [1] and [2]).

In the ordinary fixed point theory, the underlying spaces are topological spaces. The considered mappings are required to hold some continuity properties, with respect to the given topologies. In the real world, there are many problems in economics, military affairs, ecology, etc. for which the utilities cannot be totally ordered. The underlying spaces may not be equipped with a topological structure or an algebraic structure. In these cases, the traditional techniques used in totally ordered spaces endowed with topology structure cannot be appropriately applied. It leads many authors to develop some new analysis techniques in non-totally ordered spaces (see [1,3-5]). So the first important task is to develop some fixed point theorem on ordered spaces without using topological properties.

(c) $2015 \mathrm{Li}$. This article is distributed under the terms of the Creative Commons Attribution 4.0 International License (http://creativecommons.org/licenses/by/4.0/), which permits unrestricted use, distribution, and reproduction in any medium, provided you give appropriate credit to the original author(s) and the source, provide a link to the Creative Commons license, and indicate if changes were made. 
In 1955, Tarski proved a fixed point theorem on chain-complete lattices for single-valued mappings.

Tarski fixed point theorem [6] Let $(P, \succeq)$ be a chain-complete lattice and let $F: P \rightarrow P$ be an order-increasing single-valued mapping. If there is an $x^{*}$ in $P$ with $F\left(x^{*}\right) \geq x^{*}$, then $F$ has a fixed point.

It was extended to chain-complete posets in 1961.

Abian-Brown fixed point theorem [7] Let $(P, \succeq)$ be a chain-complete poset and let $F$ : $P \rightarrow P$ be an order-increasing single-valued mapping. If there is an $x^{*}$ in $P$ with $F\left(x^{*}\right) \succeq x^{*}$, then $F$ has an $\succeq$-maximum fixed point.

In 1984, Fujimoto extended Tarski fixed point theorem from single-valued mappings to set-valued mappings:

Fujimoto fixed point theorem [8] Let $(P, \succeq)$ be a complete lattice and let $F: P \rightarrow 2^{P} \backslash\{\emptyset\}$ be a set-valued map. If $F$ satisfies the following two conditions:

A1. $F$ is isotone ( $F$ is order-increasing upward).

A2. The set $\{z \in P: z \preceq u$ for some $u \in F(x)\}$ is an inductively ordered set for each $x \in P$. Then $F$ has a fixed point, that is, there exists $x^{*} \in P$ such that $x^{*} \in F\left(x^{*}\right)$.

The present author generalized the Fujimoto fixed point theorem to chain-complete posets in 2014.

Theorem 1.1 ([3], Theorems 2.2) Let $(P, \succeq)$ be a chain-complete poset and let $F: P \rightarrow$ $2^{P} \backslash\{\varnothing\}$ be a set-valued mapping satisfying the following three conditions:

A1. $F$ is order-increasing upward.

A2. $(F(x), \succeq)$ is inductive with a finite number of maximal elements, for every $x \in P$.

A3. There is an element $y$ in $P$ with $y \preceq v$, for some $v \in F(y)$.

Then F has a fixed point.

In [3] and [4], several fixed point theorems are proved for set-valued mappings on chinacomplete posets and some applications have been provided. In the theorems in [3] and [4], the condition A2 requires that the values of the considered mapping must be inductive with a finite number of maximal elements. In this paper, we first show that this condition is necessary for the considered mappings to have a fixed point. Then we will show that the set of fixed points of some order-preserving set-valued mappings is inductive, which provides some useful properties for applications. We will also develop more fixed point theorems on both partially ordered sets and partially ordered topological spaces. Moreover, we will investigate some conditions for the considered mappings to substitute the chain-completeness of the underlying spaces.

\section{Preliminaries}

In this section, we recall some concepts and properties of posets. The notations in order theory used in this paper closely follow that from [1, 3, 4, 7-9], and [10].

Let $\left(X, \succeq^{X}\right),\left(U, \succeq^{U}\right)$ be posets and $F: X \rightarrow 2^{U} \backslash\{\emptyset\}$ a set-valued mapping. $F$ is said to be isotone, or to be order-increasing upward, if $x \preceq^{X} y$ in $X$, then for any $z \in F(x)$, there is a 
$w \in F(y)$ such that $z \preceq^{U} w$. $F$ is said to be order-increasing downward, if $x \preceq^{X} y$ in $X$, then for any $w \in F(y)$, there is a $z \in F(x)$ such that $z \preceq^{U} w$. If $F$ is both order-increasing upward and order-increasing downward, then $F$ is said to be order-increasing.

As a special case, a single-valued mapping $F$ from a poset $\left(X, \succeq^{X}\right)$ to a poset $\left(U, \succeq^{U}\right)$ is said to be order-increasing whenever $x \preceq^{X} y$ implies $F(x) \preceq^{U} F(y)$. An increasing mapping $F: X \rightarrow U$ is said to be strictly order-increasing whenever $x \prec^{X} y$ implies $F(x) \prec^{U} F(y)$.

A nonempty subset $A$ of a poset $(P, \succeq)$ is said to be inductive whenever any given chain $\left\{x_{\alpha}\right\} \subseteq A$ has an upper cover in $A$. $A$ is said to be re-inductive whenever any given chain $\left\{x_{\alpha}\right\} \subseteq A$ has a lower bound in $A$. $A$ is said to be bi-inductive if and only if $A$ is both inductive and re-inductive. From Zorn's lemma and its dual version, every bi-inductive subset of a poset $(P, \succeq)$ contains an $\succeq$-minimal element and an $\succeq$-maximal element (in $A$ ). The following concept was introduced in [4] (Definition 3.1 therein).

A nonempty subset $A$ of a poset $(P, \succeq)$ is said to be universally inductive in $P$ if, for any given chain $\left\{x_{\alpha}\right\} \subseteq P$ satisfying the requirement that if every element $x_{\beta} \in\left\{x_{\alpha}\right\}$ has an upper cover in $A$, then the chain $\left\{x_{\alpha}\right\}$ has an upper bound in $A$. By applying the concept of universally inductive posets, a fixed point theorem is proved in [4].

Theorem 2.1 ([4], Theorem 3.4) Let $(P, \succeq)$ be a chain-complete poset and let $F: P \rightarrow$ $2^{P} \backslash\{\emptyset\}$ be a set-valued mapping satisfying the following three conditions:

A1. $F$ is order-increasing upward.

A2. $(F(x), \succeq)$ is universally inductive, for every $x \in P$.

A3. There is an element $y_{*}$ in $P$ and $v_{*} \in F\left(y_{*}\right)$ with $y_{*} \preceq v_{*}$.

Then $F$ has a fixed point.

The concept of universally inductive posets is more general than the concept of inductive posets with a finite number of maximal elements. It has been proved in [4].

Lemma 2.2 ([4], Lemma 3.2) Every inductive subset $A$ in a chain-complete poset with a finite number of maximal elements is universally inductive.

From Lemma 3.2 in [4], Theorems 2.2 in [3] can be immediately obtained as a corollary of Theorem 3.4 in [4]. In Theorems 2.2 in [3] listed above, the conditions A1 and A2 for the given mapping $F$ look very natural; and the condition that the poset $(P, \succeq)$ is chaincomplete and condition A2 for $F$ seem to be very strong and 'superfluous'. In fact, these conditions cannot be deleted in this theorem. We provide some counter examples below to show that in condition A2 of Theorems 2.2 [3], the condition that, for every $x \in P$, the set of the maximal elements of the inductive set $(F(x), \succeq)$ is finite is necessary for ensuring $F$ to have a fixed point.

Example 2.3 Let $S$ be the closed set in $R_{2}=\{(u, v): u, v \in R\}$ enclosed by the triangle with vertices $(1,0),(0,1)$ and $(2,2)$; and let $L$ be the closed segment in $R_{2}$ with ending points $(2,2)$ and $(4,0)$. Take $P$ to be the union of $S$ and $L$ and let $P$ be equipped with the component-wise ordering in $R_{2}$, which is denoted by $\succeq^{2}$.

One can show that $\left(P, \succeq^{2}\right)$ is not a lattice. But it is a chain-complete poset! For any given nonnegative number $a \in[0,3)$, let $S_{a}$ be the intersection of $S$ and the line $v=-u+1+a$, that is,

$$
S_{a}=S \cap\{(u,-u+1+a): u \in R\} .
$$


We have $\bigcup_{0 \leq a<3} S_{a}=S \backslash\{(2,2)\}$. It can be shown that every two distinct points in $S_{a}$ are not $\succeq^{2}$-comparable. Then $S_{a}$ is inductive and every point in $S_{a}$ is a $\succeq^{2}$-maximal point of $S_{a}$.

For any two distinct points $\left(u_{1}, v_{1}\right),\left(u_{2}, v_{2}\right) \in R_{2}$, let $\left(\left(u_{1}, v_{1}\right),\left(u_{2}, v_{2}\right)\right)$ denote the open interval in $R_{2}$ with (not included) ending points $\left(u_{1}, v_{1}\right)$ and $\left(u_{2}, v_{2}\right)$; and let $\left(\left(u_{1}, v_{1}\right),\left(u_{2}, v_{2}\right)\right]$ denote the half open interval in $R_{2}$ not including the ending point $\left(u_{1}, v_{1}\right)$ but including $\left(u_{2}, v_{2}\right)$.

We define a set-valued mapping $F: P \rightarrow 2^{P} \backslash\{\emptyset\}$ as below:

(1) For any point $(u,-u+1+a) \in S_{a}$, with a given number $a \in[0,3)$, let

$$
F(u,-u+1+a)=(1+a / 3,1+a / 3) .
$$

(2) For any point $(u,-u+4)$ on the interval $((2,2),(4,0)]$, define

$$
F(u,-u+4)=(2,2), \quad \text { for all } 2<u \leq 4 .
$$

(3) $F(2,2)=((2,2),(4,0))$.

In this example, $F$ has single value at all points except point $(2,2)$. One can check that $F$ satisfies all conditions in Theorems 2.2 [3], except the condition that the set of the maximal elements of the unique inductive set $\left(F(2,2), \succeq^{2}\right)$ is not finite. It is clear that $\mathcal{F}(F)=\emptyset$.

Furthermore, by modifying the above Example 2.3, we provide counterexamples for Theorems 2.2 [3] below with chain-complete lattices.

Example 2.4 Let $S, L$ be defined as in Example 2.3; and let $T$ be the closed triangle in $R_{2}$ with vertices $(2,2),(4,0)$ and $(4,2)$ (note that $L$ is the hypotenuse of the right triangle $T$ ). Take $P$ to be the union of $S$ and $T$ and let $P$ be equipped with the component-wise ordering $\succeq^{2}$ on $R_{2}$. One can show that $\left(P, \succeq^{2}\right)$ is a chain-complete lattice!

For any given number $b \in[2,4]$, let $T_{b}$ be the intersection of $T$ and the line $u=b$, that is,

$$
T_{b}=T \cap\{(b, v): v \in R\}=\{(b, v):-b+4 \leq v \leq 2\} .
$$

We define a set-valued mapping $F: P \rightarrow 2^{P} \backslash\{\emptyset\}$ as below:

1. For any point $(u,-u+1+a) \in S_{a}$, with a given number $a \in[0,3)$, let

$$
F(u,-u+1+a)=(1+a / 3,1+a / 3) .
$$

2. For any point $(u,-u+4)$ on the open interval $((2,2),(4,0))$ (where we must have $2<u<4)$, define

$$
F(u,-u+4)=(2,2) .
$$

3. $F(2,2)=((2,2),(4,0))$.

4. For any point $(b, v) \in T \backslash L$, with a number $b \in(2,4]$, let

$$
F(b, v)=(1+b / 2,2), \quad \text { for any }-b+4 \leq v \leq 2 .
$$


In this example, $F$ has a single value at all points except the point $(2,2)$, at which the set of the maximal elements of the inductive set $\left(F(2,2), \succeq^{2}\right)$ is not finite. One can check that, except for condition A2, $F$ satisfies all other conditions in Theorems 2.2 [3]. It is clear that $\mathcal{F}(F)=\emptyset$.

Furthermore, we will next provide a counterexample to demonstrate that if the poset $(P, \succeq)$ is not chain-complete, then a mapping $F$ satisfying conditions A1, A2, and A3 in Theorem 2.2 [3] may not have a fixed point.

Example 2.5 Take $P=[0,1) \cup(1,2]$ equipped with the ordinary order $\geq$ of real numbers. Then $(P, \geq)$ is a lattice that is not chain-complete. We define a mapping $F: P \rightarrow P$ as below:

$$
F(x)=(1+x) / 2, \quad \text { for all } x \in P .
$$

It can be checked that $F$ satisfies all conditions A1, A2, and A3 in Theorem 4.1 [3]. But $F$ does not have a fixed point.

\section{Inductive properties of fixed point sets}

In this section, we apply fixed point Theorem 3.4 in [4] to go one step further to study the inductive properties of fixed point sets of some mappings on chain-complete posets. For a set-valued mapping $F$ on a poset, let $\mathcal{F}(F)$ denote the set of fixed points of $F$.

Theorem 3.1 Let $(P, \succeq)$ be a chain-complete poset and let $F: P \rightarrow 2^{P} \backslash\{\emptyset\}$ be a set-valued mapping satisfying the following three conditions:

A1. $F$ is order-increasing upward.

A2. $(F(x), \succeq)$ is universally inductive, for every $x \in P$.

A3. There is an element $y_{*}$ in $P$ and $v_{*} \in F\left(y_{*}\right)$ with $y_{*} \preceq v_{*}$.

Then:

(i) $(\mathcal{F}(F), \succeq)$ is a nonempty inductive poset.

(ii) $\left(\mathcal{F}(F) \cap\left[y_{*}\right), \succeq\right)$ is a nonempty inductive poset; and $F$ has an $\succeq$-maximal fixed point $x^{*}$ with $x^{*} \succeq y_{*}$.

Proof At first, we prove part (i). Let

$$
A=\{z \in P \text { : there is } v \in F(z) \text { with } z \preceq v\} .
$$

It is clear that $\mathcal{F}(F) \subseteq A$. From Theorem 3.4 [4], $\mathcal{F}(F) \neq \emptyset$, and, therefore, $A \neq \emptyset$ (In fact, from another point of view, the element $y_{*}$ given in condition A3 is in $A$, which also implies that $A \neq \emptyset$.). It will next be shown that $(\mathcal{F}(F), \succeq)$ is an inductive poset. To this end, taking any arbitrary chain $\left\{x_{\alpha}\right\} \subseteq \mathcal{F}(F)$, since $(P, \succeq)$ is chain-complete, the supremum $\bigvee\left\{x_{\alpha}\right\}$ exists in $P$. We write $x=\bigvee\left\{x_{\alpha}\right\}$. Then we need to show that $\left\{x_{\alpha}\right\}$ has an upper bound in $(\mathcal{F}(F), \succeq)$.

We claim $x \in A$. Since $F$ is order-increasing upward, for any $x_{\alpha}$, from $x_{\alpha} \preceq x$, and $x_{\alpha} \in F\left(x_{\alpha}\right)$, there is an element $v_{\alpha} \in F(x)$ such that $x_{\alpha} \preceq v_{\alpha}$. By condition A2, $(F(x), \succeq)$ is universally inductive. Similarly to the proof of Theorem 3.4 in [4], we can show that there is an $\succeq$-maximal point $v \in F(x)$ such that

$$
x_{\alpha} \preceq v, \quad \text { for all } x_{\alpha} \text { in the chain }\left\{x_{\alpha}\right\} .
$$


Since $x=\bigvee\left\{x_{\alpha}\right\}$, it implies $x \preceq v \in F(x)$; and therefore $x \in A$. It follows that $[x) \cap A \neq \emptyset$, where $[x)=\{y \in P: y \succeq x\}$. Then we divide the rest of the proof into two parts:

1. Suppose $[x) \cap A=\{x\}$. From the above proof, the $\succeq$-maximal point $v \in F(x)$ satisfies $x \preceq v$. From the order-increasing upward property of $F$, for the given $v \in F(x)$ with $x \preceq v$, there is $w \in F(v)$ such that $v \preceq w$. It implies that $v \in A$. From the assumption $[x) \cap A=\{x\}(x$ is an $\succeq$-maximal element in $(A, \succeq))$ and $x \preceq v$, it follows that $x=v \in F(x)$. Hence $x \in \mathcal{F}(F)$, and, therefore, $x$ is an upper bound of the chain $\left\{x_{\alpha}\right\}$ in $\mathcal{F}(F)$.

2. Suppose $[x) \cap A$ is not a singleton. Then $([x) \cap A, \succeq)$ is also a poset. From the Hausdorff maximality theorem, $([x) \cap A, \succeq)$ contains a maximal chain $\left\{x_{\beta}\right\}$ in $([x) \cap A, \succeq)$ (with respect to the sets inclusion partial order $\supseteq$ ). Since $x$ is the smallest element in $[x] \cap A$, it implies that $\left\{x_{\beta}\right\}$ contains $x$ as its smallest element. So $\left\{x_{\alpha}\right\} \cup\left\{x_{\beta}\right\}$ is a chain in $(P, \succeq)$. Then $\bigvee\left(\left\{x_{\alpha}\right\} \cup\left\{x_{\beta}\right\}\right)$ exists in $P$, which is denoted by $y=\bigvee\left(\left\{x_{\alpha}\right\} \cup\left\{x_{\beta}\right\}\right)$. Since $\left\{x_{\alpha}\right\} \subseteq \mathcal{F}(F)$, for every index $\alpha$, by condition A1, as showed in the above proof, there is $v_{\alpha} \in F(y)$ such that $x_{\alpha} \preceq v_{\alpha}$. From $\left\{x_{\beta}\right\} \subseteq A$, for every $\beta$, there is $u_{\beta} \in F\left(x_{\beta}\right)$ such that $x_{\beta} \preceq u_{\beta}$. From the order-increasing upward property of $F$, there is $v_{\beta} \in F(y)$ such that $x_{\beta} \preceq u_{\beta} \preceq v_{\beta}$. By condition $\mathrm{A} 2,(F(y), \succeq)$ is universally inductive, and, therefore, there is an $\succeq$-maximal point $u \in F(y)$ such that

$x_{\alpha} \preceq u$, and $x_{\beta} \preceq u$, for all $x_{\alpha}$ in the chain $\left\{x_{\alpha}\right\}$ and for all $x_{\beta}$ in the chain $\left\{x_{\beta}\right\}$.

Since $y=\bigvee\left(\left\{x_{\alpha}\right\} \cup\left\{x_{\beta}\right\}\right)$, it implies $y \preceq u \in F(y)$, which implies that $y \in A$. From $x \preceq y$, it follows that $y \in[x) \cap A$. Since $\left\{x_{\beta}\right\}$ is a maximal chain $\left\{x_{\beta}\right\}$ in $([x) \cap A, \succeq)$, we must have $y \in\left\{x_{\beta}\right\}$.

On the other hand, from the order-increasing upward property of $F$, from $y \preceq u \in F(y)$, there is $w \in F(u)$ such that $u \preceq w$. So we have $u \in A$. From $x \preceq y \preceq u \preceq w$, it implies that $u \in[x) \cap A$. Since $\left\{x_{\beta}\right\}$ is a maximal chain in $([x) \cap A, \succeq)$ and $y=\bigvee\left(\left\{x_{\alpha}\right\} \cup\left\{x_{\beta}\right\}\right) \in\left\{x_{\beta}\right\}$, by $y \preceq u \in[x) \cap A$, it follows that $u=y$. It implies that $y \in \mathcal{F}(F)$. From $y=\bigvee\left(\left\{x_{\alpha}\right\} \cup\left\{x_{\beta}\right\}\right)$, it shows that $y$ is an upper bound of the chain $\left\{x_{\alpha}\right\}$ in $\mathcal{F}(F)$.

Hence in either case, we proved that the arbitrary chain $\left\{x_{\alpha}\right\} \subseteq \mathcal{F}(F)$ has an upper bound in $(\mathcal{F}(F), \succeq)$. It follows that $(\mathcal{F}(F), \succeq)$ is inductive.

Then we prove part (ii). From condition A3, $v_{*} \in\left[y_{*}\right.$ ), and, therefore, $\left[y_{*}\right) \neq \emptyset$. It is clear that $y_{*} \in\left[y_{*}\right) \cap A$. By the increasing upward property of $F$, we can show that $v_{*} \in\left[y_{*}\right) \cap$ $A$. From the Hausdorff maximality theorem, $\left(\left[y_{*}\right) \cap A, \succeq\right)$ contains a maximal chain $\left\{x_{\gamma}\right\}$ containing $y_{*}$ as its smallest element. Since $(P, \succeq)$ is chain complete, there exists $z=\bigvee\left\{x_{\gamma}\right\}$. Similarly to the proof of step 2 above, we can show that $z \in \mathcal{F}(F)$. From $z=\bigvee\left\{x_{\gamma}\right\} \succeq y_{*}$, it follows that $z \in \mathcal{F}(F) \cap\left[y_{*}\right)$. Hence $\mathcal{F}(F) \cap\left[y_{*}\right) \neq \emptyset$. From part (i), $\mathcal{F}(F)$ is inductive and as an order-interval of a chain-complete poset, $\left[y_{*}\right)$ is also inductive. It implies that $\mathcal{F}(F) \cap\left[y_{*}\right)$ is a nonempty inductive subset of $(P, \succeq)$.

By Lemma 3.2 in [4], as a consequence of Theorem 3.1, we have the following result. Since it is important for application, we state it with full details as a corollary.

Corollary 3.2 Let $(P, \succeq)$ be a chain-complete poset and let $F: P \rightarrow 2^{P} \backslash\{\emptyset\}$ be a set-valued mapping satisfying the following three conditions:

A1. $F$ is order-increasing upward.

A2. $(F(x), \succeq)$ is inductive with a finite number of maximal elements, for every $x \in P$. 
A3. There is an element $y_{*}$ in $P$ and $v_{*} \in F\left(y_{*}\right)$ with $y_{*} \preceq v_{*}$.

Then:

(i) $(\mathcal{F}(F), \succeq)$ is a nonempty inductive poset.

(ii) $\left(\mathcal{F}(F) \cap\left[y_{*}\right), \succeq\right)$ is a nonempty inductive poset, and $F$ has an $\succeq$-maximal fixed point $x^{*}$ with $x^{*} \succeq y_{*}$.

In the applications of fixed point theory, there are many cases that the considered underlying spaces are not chain-complete. So it is worthy to find some other conditions to substitute the chain-completeness in Theorem 3.1. In the following theorem, the chaincompleteness condition for the underlying space is replaced by the chain-completeness property of the range of the considered mapping, where the range of a set-valued mapping $F: P \rightarrow 2^{P} \backslash\{\emptyset\}$ is defined as

$$
F(P)=\bigcup\{F(x): x \in P\}
$$

Theorem 3.3 Let $(P, \succeq)$ be a poset and let $F: P \rightarrow 2^{P} \backslash\{\emptyset\}$ be a set-valued mapping satisfying the following four conditions:

A1. $F$ is $\succeq$-increasing upward.

A2. $(F(x), \succeq)$ is universally inductive, for every $x \in P$.

A3. There is an element $y_{*}$ in $P$ and $v_{*} \in F\left(y_{*}\right)$ with $y_{*} \preceq v_{*}$.

A4. The range $(F(P), \succeq)$ is a chain-complete poset.

Then:

(i) $(\mathcal{F}(F), \succeq)$ is a nonempty inductive poset.

(ii) $\left(\mathcal{F}(F) \cap\left[y_{*}\right), \succeq\right)$ is a nonempty inductive poset, and $F$ has an $\succeq$-maximal fixed point $x^{*}$ with $x^{*} \succeq y_{*}$.

Proof For the given mapping $F$, let $F_{R}$ denote the restriction mapping of $F$ on the range $F(P)$. It is clear to see that $F_{R}: F(P) \rightarrow 2^{F(P)} \backslash\{\emptyset\}$ is a well-defined set-valued mapping. We have

$$
\mathcal{F}(F) \subseteq F(P) .
$$

Notice that, for every $x \in F(P), F_{R}(x)=F(x)$. It implies

$$
\mathcal{F}\left(F_{R}\right)=\mathscr{F}(F) .
$$

From the conditions A1 and A2 for the mapping $F$ in this theorem, it immediately follows that $F_{R}$ satisfies the conditions $\mathrm{A} 1$ and $\mathrm{A} 2$ of Theorem 3.1 on $(F(P), \succeq)$.

For the elements $y_{*}, v_{*}$ given in condition A3 in this theorem, since $v_{*} \in F\left(y_{*}\right)$ and $y_{*} \preceq$ $v_{*}$, from the $\succeq$-increasing upward property of $F$, there is $u_{*} \in F\left(v_{*}\right)$ with $v_{*} \preceq u_{*}$. From $v_{*} \in F(P)$, it implies that $u_{*} \in F_{R}\left(v_{*}\right)$ satisfying $v_{*} \preceq u_{*}$. Hence $F_{R}$ satisfies condition A3 in Theorem 3.1 on $(F(P)$, $)$ with the elements $v_{*}, u_{*} \in F(P)$ such that $u_{*} \in F_{R}\left(v_{*}\right)$ and $v_{*} \preceq u_{*}$. From condition $\mathrm{A} 4,(F(P), \succeq)$ is a chain-complete poset. Hence by applying Theorem 3.1, we obtain:

1. $\left(\mathcal{F}\left(F_{R}\right), \succeq\right)$ is a nonempty inductive poset, and, therefore, $F$ has an $\succeq$-maximal fixed point. 
2. $\left(\mathcal{F}\left(F_{R}\right) \cap\left[y_{*}\right) \cap F(P), \succeq\right)$ is a nonempty inductive poset, and, therefore, $F$ has an $\succeq$-maximal fixed point $x^{*}$ with $x^{*} \succeq y_{*}$.

By (2), we have $(\mathcal{F}(F), \succeq)=\left(\mathcal{F}\left(F_{R}\right), \succeq\right)$. By (2) and (1), we get

$$
\left(\mathcal{F}\left(F_{R}\right) \cap\left[y_{*}\right) \cap F(P), \succeq\right)=\left(\mathcal{F}(F) \cap\left[y_{*}\right), \succeq\right)
$$

Then this theorem immediately follows from the above results 1 and 2 .

Similarly to Corollary 3.2, we can apply Lemma 3.2 in [4] to get the following corollary of Theorem 3.3.

Corollary 3.4 Let $(P, \succeq)$ be a poset and let $F: P \rightarrow 2^{P} \backslash\{\emptyset\}$ be a set-valued mapping satisfying the following four conditions:

A1. $F$ is $\succeq$-increasing upward.

A2. $(F(x), \succeq)$ is inductive with a finite number of maximal elements, for every $x \in P$.

A3. There is an element $y_{*}$ in $P$ and $\nu_{*} \in F\left(y_{*}\right)$ with $y_{*} \preceq v_{*}$.

A4. The range $(F(P), \succeq)$ is a chain-complete poset.

Then:

(i) $(\mathcal{F}(F), \succeq)$ is a nonempty inductive poset.

(ii) $\left(\mathcal{F}(F) \cap\left[y_{*}\right)\right.$, $)$ is a nonempty inductive poset, and $F$ has an $\succeq$-maximal fixed point $x^{*}$ with $x^{*} \succeq y_{*}$.

Let $(P, \succeq)$ be a chain-complete poset and let $F: P \rightarrow 2^{P} \backslash\{\emptyset\}$ be a set-valued mapping. We provide a counter example below to show that the two conditions A1 and A2 for $F$ given in Theorem 3.1 does not guarantee the range $(F(P), \succeq)$ to be a chain-complete poset contained in $(P, \succeq)$.

Example 3.5 Let $([0,2]$, $\geq$ ) be the (chain) complete poset (lattice) with respect to the ordinary order of real numbers. Define a set-valued mapping $F:[0,2] \rightarrow 2^{[0,2]} \backslash\{\varnothing\}$ by

$$
\begin{aligned}
& F(x)=[0, x], \quad \text { if } x \in[0,1) ; \\
& F(x)=(1,2], \quad \text { if } x \in[1,2] .
\end{aligned}
$$

It is clear to see that $F$ satisfies the conditions A1 and A2 given in Theorem 3.1. But the range of $F,(F([0,2]), \geq)=([0,1) \cup(1,2], \geq)$ is not a chain-complete poset contained in $([0,2], \geq)$.

\section{The reversed orders of partial orders}

Let $(P, \succeq)$ be a poset. Define the reversed ordering relation $\succeq^{-}$of the partial order $\succeq$ on $P$ by, for $x, y \in P$,

$$
x \succeq^{-} y \text { if and only if } x \preceq y \text {. }
$$

Then $\succeq^{-}$is also a partial order on $P$ and it is called the reversed order of $\succeq$. A poset $(P, \succeq)$ is said to be reversed chain complete, simply denoted by re-chain complete, if every chain in $P$ has an infimum (with respect to the order $\succeq$ ). There are some connections between the original partial order $\succeq$ and its reversed order $\succeq^{-}$: 
1. $(P, \succeq)$ is re-inductive, if and only if $\left(P, \succeq^{-}\right)$is inductive.

2. $(P, \succeq)$ is re-chain complete if and only if $\left(P, \succeq^{-}\right)$is chain complete.

3. A mapping $F: P \rightarrow 2^{P} \backslash\{\emptyset\}$ is $\succeq$-increasing downward, if and only if it is $\succeq^{-}$-increasing upward.

The reversal version of Definition 3.1 in [4] is that a nonempty subset $A$ of a poset $(P, \succeq)$ is said to be universally re-inductive in $P$ if, for any given chain $\left\{x_{\alpha}\right\} \subseteq P$ satisfying the requirement that every element $x_{\beta} \in\left\{x_{\alpha}\right\}$ has a lower bound in $A$, the chain $\left\{x_{\alpha}\right\}$ has a lower bound in $A$.

The dual version of Lemma 3.2 in [4] is that every inductive subset $A$ in a poset satisfying that $A$ has a finite number of minimal elements is universally re-inductive. Under the above definitions, the results in the previous section can easily be adapted to the cases of the reversed order with some suitable modifications. We list the dual versions of Theorem 3.1 as theorems below.

Theorem 4.1 Let $(P, \succeq)$ be a re-chain-complete poset and let $F: P \rightarrow 2^{P} \backslash\{\emptyset\}$ be a setvalued mapping satisfying the following three conditions:

A1. $F$ is order-increasing downward.

A2. $(F(x), \succeq)$ is universally re-inductive, for every $x \in P$.

A3. There is an element $y^{*}$ in $P$ and $v^{*} \in F\left(y^{*}\right)$ with $v^{*} \preceq y^{*}$.

Then:

(i) $(\mathcal{F}(F), \succeq)$ is a nonempty re-inductive poset.

(ii) $\left(\mathcal{F}(F) \cap\left(y^{*}\right], \succeq\right)$ is nonempty and re-inductive, and $F$ has an $\succeq$-minimal fixed point $x^{*}$ with $x^{*} \preceq y^{*}$.

The inductive property and the order-maximal of the set of fixed points are studied in Theorem 3.1. By applying the reversed order, the re-inductive property and the orderminimal points of the set of fixed points are studied in Theorem 4.1. By combining these two theorems, we can derive the following result dealing with bi-inductive properties of the set of fixed points.

Theorem 4.2 Let $(P, \succeq)$ be a chain-complete and re-chain-complete poset. Let $F: P \rightarrow$ $2^{P} \backslash\{\emptyset\}$ be a set-valued mapping satisfying the following three conditions:

A1. $F$ is $\succeq$-increasing.

A2. $(F(x), \succeq)$ is both universally inductive and universally re-inductive, for every $x \in P$.

A3. There are elements $y^{*}, z^{*}$ in $P$ with $y^{*} \preceq z^{*}$ satisfying $F\left(y^{*}\right) \cap\left[y^{*}\right) \neq \emptyset$ and $F\left(z^{*}\right) \cap\left(z^{*}\right] \neq \emptyset$.

Then:

(i) $(\mathcal{F}(F), \succeq)$ is a nonempty bi-inductive poset.

(ii) $\left(\mathscr{F}(F) \cap\left[y^{*}\right), \succeq\right)$ is a nonempty inductive poset.

(iii) $\left(\mathcal{F}(F) \cap\left(z^{*}\right], \succeq\right)$ is a nonempty re-inductive poset.

Moreover, if, in addition to conditions $\mathrm{A} 1$ and $\mathrm{A} 2$ given above, $F$ satisfies

$\mathrm{A} 3^{\prime}$. There are elements $y^{*}, z^{*}$ in $P$ with $y^{*} \preceq z^{*}$ satisfying $F\left(y^{*}\right) \subseteq\left[y^{*}, z^{*}\right]$ and $F\left(z^{*}\right) \subseteq$ $\left[y^{*}, z^{*}\right]$.

Then we have

(iv) $\left(\mathcal{F}(F) \cap\left[y^{*}, z^{*}\right], \succeq\right)$ is a nonempty bi-inductive poset; and $F$ has a fixed point $x^{*}$ with $y^{*} \preceq x^{*} \preceq z^{*}$. 
Proof We only need to prove part (iv). It is clear that $\left(\left[y^{*}, z^{*}\right], \succeq\right)$ is also a chain-complete and re-chain-complete poset. First, we show that, for any $x \in\left[y^{*}, z^{*}\right], F(x) \subseteq\left[y^{*}, z^{*}\right]$. To this end, take an arbitrary $w \in F(x), y^{*} \preceq x$ and $x \succeq z^{*}$, the $\succeq$-increasing property of $F$ implies that there are elements $v \in F\left(y^{*}\right)$ and $u \in F\left(z^{*}\right)$ such that $v \preceq w \preceq u$. Since $u, v \in\left[y^{*}, z^{*}\right]$, it follows that $w \in\left[y^{*}, z^{*}\right]$. It implies that the restriction of $F:\left[y^{*}, z^{*}\right] \rightarrow 2^{\left[y^{*}, z^{*}\right]} \backslash\{\emptyset\}$ is well defined. Then this theorem immediately follows from Theorems 3.1 and 3.3.

Theorems 4.1 and 4.2 still hold if condition A2 is modified as given in the following theorem.

Theorem 4.3 Let $(P, \succeq)$ be a chain-complete and re-chain-complete poset. Let $F: P \rightarrow$ $2^{P} \backslash\{\emptyset\}$ be a set-valued mapping satisfying the following three conditions:

A1. $F$ is $\succeq$-increasing.

A2. $(F(x), \succeq)$ is bi-inductive with a finite number of maximal elements and a finite number of minimal elements, for every $x \in P$.

A3. There are elements $y^{*}, z^{*}$ in $P$ with $y^{*} \preceq z^{*}$ satisfying $F\left(y^{*}\right) \cap\left[y^{*}\right) \neq \emptyset$ and $F\left(z^{*}\right) \cap\left(z^{*}\right] \neq \emptyset$.

Then:

(i) $(\mathcal{F}(F), \succeq)$ is a nonempty bi-inductive poset.

(ii) $\left(\mathscr{F}(F) \cap\left[y^{*}\right), \succeq\right)$ is a nonempty inductive poset.

(iii) $\left(\mathcal{F}(F) \cap\left(z^{*}\right], \succeq\right)$ is a nonempty re-inductive poset.

\section{Several fixed point theorems on partially ordered topological spaces}

In many application problems, the underlying spaces are partially ordered topological spaces, which are equipped with both topology structures and ordering relations, such as Banach lattices, Riesz spaces (see $[1,6,10]$ ). In this section, we particularly examine the inductive properties of mappings on partially ordered topological spaces and partially ordered topological vector spaces.

Let $(X, \succeq)$ be a poset. For any $u, w \in X$, we recall the following $\succeq$-intervals:

$$
\begin{aligned}
& {[u)=\{x \in X: x \succeq u\}, \quad(w]=\{x \in X: x \preceq w\} \quad \text { and }} \\
& {[u, w]=[u) \cap(w]=\{x \in X: u \preceq x \preceq w\} .}
\end{aligned}
$$

Let $(X, \succeq)$ be a poset equipped with a topology $\tau$ (it is also a topological space). The topology $\tau$ is called a natural topology on $(X, \succeq)$ with respect to the partial order $\succeq$, whenever, for every $u \in P$, the $\succeq$-intervals $[u)$ and $(u]$ are all $\tau$-closed. A poset $(X, \succeq)$ equipped with a natural topology $\tau$ with respect to $\succeq$ on $X$ is called a partially ordered topological space; and it is denoted by $(X, \tau, \succeq)$.

A real vector space $X$ endowed with a partial order $\succeq$ is called a partially ordered vector space, which is written as $(X, \succeq)$ (it is a poset), if the following (order-linearity) properties hold:

1. $x \succeq y$ implies $x+z \succeq y+z$, for all $x, y, z \in X$,
2. $x \succeq y$ implies $\alpha x \succeq \alpha y$, for all $x, y \in X$ and $\alpha \geq 0$.

A partially ordered topological vector space is both a partially ordered topological space and a partially ordered vector space. 
Remark 5.1 In this paper, a topological space equipped with a partial order is called a partially ordered topological space, if the topology on this space is natural with respect to the given partial order. Particularly, a Banach space equipped with a partial order is called a partially ordered Banach space if the norm of this space induces a natural topology with respect to the given partial order, which satisfies the order-linearity properties (3). For example, it is well known that the norm topology of a Banach lattice is always natural with respect to the given lattice order. Hence any Banach lattice can be considered as a special case of partially ordered Banach spaces.

Notice that the topology $\tau$ on a topology space $X$ is natural with respect to a partial order $\succeq$ on $X$ if and only if $\tau$ is natural with respect to the reversed order $\succeq^{-}$on $X$. Then Theorem 2.3 in [4] can be immediately extended as follows.

Lemma 5.2 Let $(X, \tau, \succeq)$ be a partially ordered Hausdorff topological space. Then every nonempty compact subset of $X$ is both chain complete and re-chain complete.

In the proof of Theorem 3.1, the condition that the values of the considered mapping are universally inductive is important for the existence of fixed point. As examples, we showed that every nonempty inductive subset with a finite number of maximal elements in a poset is universally inductive. Similarly to Lemma 5.2, we extend Lemma 3.6 in [4] to provide more examples of universally inductive, which are useful in order theory with partially ordered Hausdorff topological spaces.

Lemma 5.3 Every nonempty compact subset of a partially ordered Hausdorff topological space is both universally inductive and universally re-inductive.

As a consequence of Lemma 5.3, we have the result below, which is more practical in partially ordered reflexive Banach spaces.

Lemma 5.4 Every nonempty bounded closed and convex subset of a partially ordered reflexive Banach space is both universally inductive and universally re-inductive.

Proof Since every nonempty bounded closed and convex subset of a reflexive Banach space is weakly compact, and in a partially ordered Banach space, the norm topology is natural with respect to the given partial order if and only if the weak topology is natural with respect to the same partial order, this corollary follows from Lemma 5.3 immediately.

We provide the following example to demonstrate that the concept of universally inductive is indeed broader than the concept of inductive with a finite number of maximal elements.

Example 5.5 Let $\left(R_{2}, \succeq_{2}\right)$ denote the 2-d Hilbert lattice equipped with the component partial (lattice) order $\succeq_{2}$. That is, for any $\left(x_{1}, y_{1}\right),\left(x_{2}, y_{2}\right) \in R_{2}$,

$$
\left(x_{2}, y_{2}\right) \succeq_{2}\left(x_{1}, y_{1}\right) \quad \text { if and only if } \quad x_{2} \geq x_{1} \text { and } y_{2} \geq y_{1} \text {. }
$$


Then $\left(R_{2}, \succeq_{2}\right)$ is a partially ordered topological vector spaces and the topology of induced by the Hilbert norm on $R_{2}$ is natural with respect to the partial order $\succeq_{2}$. Take $K$ to be the closed right triangle in $\left(R_{2}, \succeq_{2}\right)$ with vertices $(0,0),(0,1)$ and $(1,0)$. As a compact subposet of $\left(R_{2}, \succeq_{2}\right)$, from Lemma $5.3, K$ is universally inductive. Since $K$ is chain complete, it is inductive. But $K$ has infinitely many $\succeq_{2}$-maximal points (every point on the hypotenuse is an $\succeq_{2}$-maximal point).

From Remark 5.1 and Lemma 5.2, as a consequence of Theorem 3.1, we have the following result on a partially ordered compact Hausdorff topological space.

Theorem 5.6 Let $(X, \tau, \succeq)$ be a partially ordered compact Hausdorff topological space. Let $F: X \rightarrow 2^{X} \backslash\{\emptyset\}$ be a set-valued mapping satisfying the following three conditions:

A1. $F$ is $\succeq$-increasing upward.

A2. $F(x)$ is a $\tau$-compact subset, for every $x \in X$.

A3. There is an element $y_{*}$ in $X$ and $v_{*} \in F\left(y_{*}\right)$ with $y_{*} \preceq v_{*}$.

Then:

(i) $(\mathcal{F}(F), \succeq)$ is a nonempty inductive poset.

(ii) $\left(\mathcal{F}(F) \cap\left[y_{*}\right), \succeq\right)$ is a nonempty inductive poset, and $F$ has an $\succeq$-maximal fixed point $x^{*}$ with $x^{*} \succeq y_{*}$.

Remark 5.7 The compactness of the partially ordered Hausdorff topological space $(X, \tau, \succeq)$ given in Theorem 5.6 can be replaced by

The range $(F(X), \succeq)$ is a $\tau$-compact subset contained in $(X, \succeq)$.

By Theorem 4.2, we can obtain the useful dual versions of Theorem 5.6.

Theorem 5.8 Let $(X, \tau, \succeq)$ be a partially ordered compact Hausdorff topological space. Let $F: X \rightarrow 2^{X} \backslash\{\emptyset\}$ be a set-valued mapping satisfying the following three conditions:

A1. $F$ is $\succeq$-increasing.

A2. $F(x)$ is a $\tau$-compact subset, for every $x \in X$.

A3. There are element $y_{*}, z_{*}$ in $X$ and $v_{*} \in F\left(y_{*}\right), u_{*} \in F\left(z_{*}\right)$ with $y_{*} \preceq v_{*}$ and $z_{*} \succeq u_{*}$.

Then:

(i) $(\mathcal{F}(F), \succeq)$ is a nonempty bi-inductive poset.

(ii) $\left(\mathcal{F}(F) \cap\left[y_{*}\right), \succeq\right)$ is a nonempty inductive poset.

(iii) $\left(\mathcal{F}(F) \cap\left(z_{*}\right], \succeq\right)$ is a nonempty re-inductive poset.

Moreover, if, in addition to conditions $\mathrm{A} 1$ and $\mathrm{A} 2$ given above, $F$ satisfies

A3'. There are elements $y^{*}, z^{*}$ in $X$ with $y^{*} \preceq z^{*}$ satisfying $F\left(y^{*}\right) \subseteq\left[y^{*}, z^{*}\right]$ and $F\left(z^{*}\right) \subseteq$ $\left[y^{*}, z^{*}\right]$.

Then we have

(iv) $\left(\mathcal{F}(F) \cap\left[y^{*}, z^{*}\right], \succeq\right)$ is a nonempty bi-inductive poset; and $F$ has a fixed point $x^{*}$ with $y^{*} \preceq x^{*} \preceq z^{*}$.

Similarly to Example 5.5, we provide a counterexample below to show that, for the given mapping $F$, neither the conditions in Theorem 5.6, nor the conditions in Theorem 5.8, guarantee the set of fixed points of $F$ to be chain complete. 
Example 5.9 Let $([0,3], \geq)$ be the (chain) complete poset (lattice) with respect to the ordinary order of real numbers. Define a set-valued mapping $F:[0,2] \rightarrow 2^{[0,2]} \backslash\{\emptyset\}$ by

$$
\begin{aligned}
& F(x)=[x, x+1 / 2], \quad \text { if } x \in[0,1) ; \\
& F(x)=[1+x / 2,3], \quad \text { if } x \in[1,2) ; \\
& F(2)=[2.5,3] ; \\
& F(x)=[x, 3], \quad \text { if } x \in(2,3] .
\end{aligned}
$$

It is clear to see that $F([0,2])=[0,2]$ and $F$ satisfies all conditions A1, A2, A3 and A4 given in Theorem 5.6, and $F$ also satisfies all conditions A1, A2, A3 given in Corollary 3.4. One can check that $\mathscr{F}(F)=[0,1) \cup(2,3]$. It is inductive, but not chain complete.

From Lemmas 5.3 and 5.4, as a consequence of Theorem 5.6, we have the following fixed point theorem on partially ordered reflexive Banach spaces.

Theorem 5.10 Let $(B, \succeq)$ be a partially ordered reflexive Banach space and $X$ a bounded closed convex subset of B. Let $F: X \rightarrow 2^{X} \backslash\{\emptyset\}$ be a set-valued mapping satisfying the following three conditions:

A1. $F$ is $\succeq$-increasing.

A2. $F(x)$ is a closed and convex subset of $X$, for every $x \in X$.

A3. There are elements $y_{*}, z_{*}$ in $X$ and $v_{*} \in F\left(y_{*}\right), u_{*} \in F\left(z_{*}\right)$ with $y_{*} \preceq v_{*}$ and $z_{*} \succeq u_{*}$.

Then:

(i) $(\mathcal{F}(F), \succeq)$ is a nonempty bi-inductive poset.

(ii) $\left(\mathcal{F}(F) \cap\left[y_{*}\right), \succeq\right)$ is a nonempty inductive poset.

(iii) $\left(\mathcal{F}(F) \cap\left(z_{*}\right], \succeq\right)$ is a nonempty re-inductive poset.

Moreover, if, in addition to conditions $\mathrm{A} 1$ and $\mathrm{A} 2$ given above, $F$ satisfies

$\mathrm{A} 3^{\prime}$. There are elements $y^{*}, z^{*}$ in $X$ with $y^{*} \preceq z^{*}$ satisfying $F\left(y^{*}\right) \subseteq\left[y^{*}, z^{*}\right]$ and $F\left(z^{*}\right) \subseteq$ $\left[y^{*}, z^{*}\right]$.

Then we have

(iv) $\left(\mathcal{F}(F) \cap\left[y^{*}, z^{*}\right], \succeq\right)$ is a nonempty bi-inductive poset; and $F$ has a fixed point $x^{*}$ with $y^{*} \preceq x^{*} \preceq z^{*}$.

Recall that, in every Banach lattice, the topology (norm) is always natural with respect to the given lattice order. Hence, in Theorem 5.10, the partially ordered reflexive Banach space $(B, \succeq)$ can be substituted by a reflexive Banach lattice, as it is a special case.

\section{Competing interests}

The author declares that he has no competing interests.

\footnotetext{
Acknowledgements

The author is very grateful to the anonymous reviewers for their valuable suggestions, which improved the presentation of this paper.

Received: 15 August 2015 Accepted: 6 November 2015 Published online: 18 November 2015

References

1. Carl, S, Heikkilä, S: Fixed Point Theory in Ordered Sets and Applications: From Differential and Integral Equations to Game Theory. Springer, New York (2010)
} 
2. Gnana Bhaskar, T, Lakshmikantham, V: Fixed point theorems in partially ordered metric spaces and applications. Nonlinear Anal. 65, 1379-1393 (2006)

3. Li, JL: Several extensions of the Abian-Brown fixed point theorem and their applications to extended and generalized Nash equilibria on chain-complete posets. J. Math. Anal. Appl. 409, 1084-1092 (2014)

4. Li, JL: Fixed point theorems on partially ordered topological vector spaces and their applications to equilibrium problems with incomplete preferences. Fixed Point Theory Appl. 2014, 192 (2014)

5. Li, JL: Existence of continuous solutions of nonlinear Hammerstein integral equations proved by fixed point theorems on posets. J. Nonlinear Convex Anal. (2015, to appear)

6. Tarski, A: A lattice-theoretical fixed point theorem and its applications. Pac. J. Math. 5, $285-309$ (1955)

7. Abian, S, Brown, A: A theorem on partially ordered sets with applications to fixed point theorem. Can. J. Math. 13, 78-83 (1961)

8. Fujimoto, T: An extension of Tarski's fixed point theorem and its applications to isotone complementarity problems. Math. Program. 28, 116-118 (1984)

9. Ok, EA: Order Theory (forthcoming)

10. Ward, LE Jr.: Partially ordered topological space. Proc. Am. Math. Soc. 5(1), 144-161 (1954)

Submit your manuscript to a SpringerOpen ${ }^{\odot}$ journal and benefit from:

- Convenient online submission

Rigorous peer review

- Immediate publication on acceptance

- Open access: articles freely available online

- High visibility within the field

- Retaining the copyright to your article 